\title{
RECOVERY OF CTF BEAM SIGNALS FROM A STRONG WAKEFIELD BACKGROUND
}

\author{
Yan Yin, TRIUMF, 4004 Wesbrook Mall, Vancouver, B.C. V6T 2A3, Canada \\ Elmar Schulte, CERN, CH-1211 Geneva 23, Switzerland \\ Tord Ekelöf, Uppsala University, P.O.Box 535, S-751 21, Sweden
}

\begin{abstract}
The beam monitor for the CERN Linear Collider Test Facility (CTF) has to work not only with very short pulses (3$50 \mathrm{ps}$ FWHM) at a spacing of $330 \mathrm{ps}$, but also in a strong wakefield background. A cone-shaped button pickup electrode has been designed and constructed for use with CTF beams and tests have been made using a real time analogue Gaussian filter to recover the beam signals from the strong wakefield signals. As a comparison to the analogue filter, a study has been made to process the data off-line and extract the beam signals using digital filtering based on the wavelet concept.
\end{abstract}

\section{INTRODUCTION}

The CERN Linear Collider Test Facility (CTF) is a $4.5-45$ $\mathrm{MeV}$ linac producing an intense electron beam with the following parameters:

Number of bunches in the train:

Bunch length (FWHM):

$1-24$

Bunch repetition rate in the train:

3-50 ps

$3 \mathrm{GHz}$

$10 \mathrm{~Hz}$

Train repetition rate:

$40.5 \mathrm{~mm}$

Normally, the bunch spacing is 333 ps and bunch length $\leq 12$ ps FWHM. Each bunch contains $5.6 \times 10^{10}$ electrons, that is a charge of $9 \mathrm{nC}$. Assuming the bunches have a Gaussian longitudinal distribution, it follows that when rms HW $\sigma$ is $25 \mathrm{ps}$, the peak current is $144 \mathrm{~A}$ and when $\sigma$ is $6 \mathrm{ps,}$ the peak current is $598 \mathrm{~A}^{[1]}$.

The CTF beam monitor is intended to measure the intensity and transverse position of each bunch. When the pickup geometric size is comparable to the bunch length, the longitudinal intensity distribution can be recovered by integrating the pickup signal. However, because strong wakefields are present in the CTF, the first task is to extract the beam signal from the wakefield background.

An ordinary button-type electrode senses the signal across the capacitance between the button and the wall. A resonance may occur if the structure of the button and its housing form a cavity. When the bunch length is much longer than the button size, the beam frequency spectrum does not extend up to the resonance frequency, and the resonance is not excited. However, when the bunch length is comparable to the button size, the resonance may be excited, and if it is within the frequency of interest, it may disturb observation of the beam signal. Therefore the pickup has to be carefully designed to avoid such parasitic resonances
A cone-shaped button pickup has been designed to reach this goal; Fig. 1 shows the geometry. The pickup electrode is made with a coaxial cone shape and is directly welded into a Kaman feedthrough, which is useable up to $40 \mathrm{GHz}$. The cone shaped pickup has a smooth transition to the feedthrough. The ratio of the cone radius, $a$, to the outer conductor radius, $b$, is kept constant at $b / a=2.3$ along the cone axis in order to keep the impedance constant at $50 \Omega$. In Fig. $1, a=1.8 \mathrm{~mm}, b=3.9$ $\mathrm{mm}, a^{\prime}=0.79 \mathrm{~mm} b^{\prime}=1.45 \mathrm{~mm}$ (Kaman feedthrough), and the height of the cone is $4 \mathrm{~mm}$.

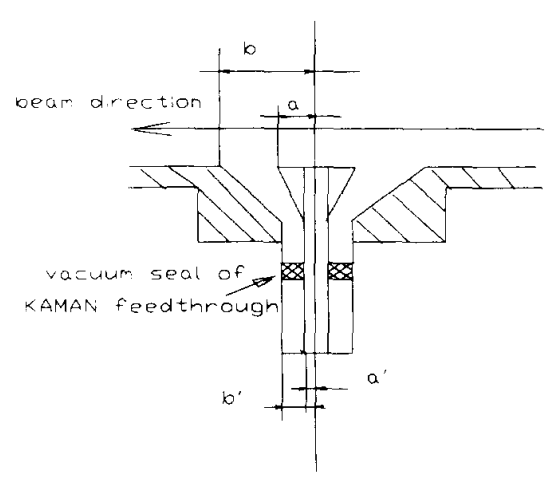

Fig.1 Cross-section of the cone-shaped button pickup.
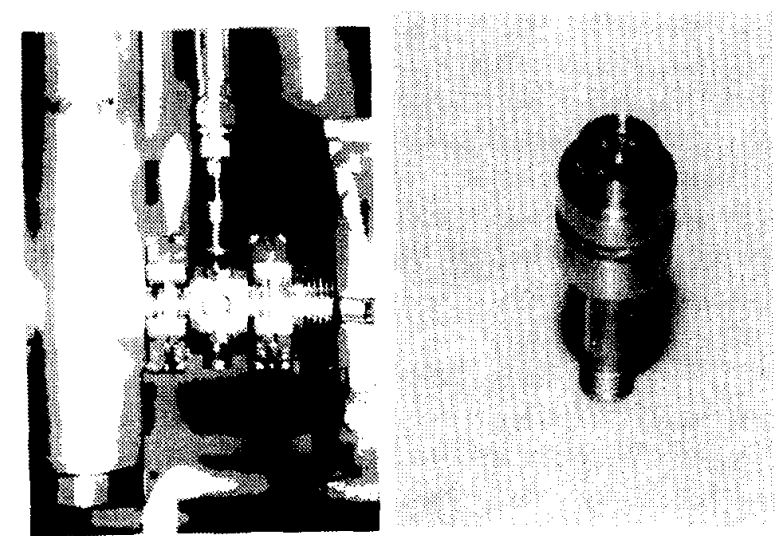

Fig. 2 The cone-shaped pickup installed in the CTF (left) and a pickup before being welded into the beam pipe (right).

Two prototype monitors have been made and tested at the CTF. The design calculations and test results have been reported elsewhere ${ }^{[1]}$. $S_{11}$ measurements have been made in the time domain to study the resonances of the pickup. Fig.3 
shows the synthesized incident pulse at $40 \mathrm{GHz}(25 \mathrm{ps}$ FWHM), which is the frequency limit of the network analyzer HP8510, and the reflection from the pickup. The pickup has small resonances at $15 \mathrm{GHz}$ with less than $5 \%$ of the original signal amplitude.

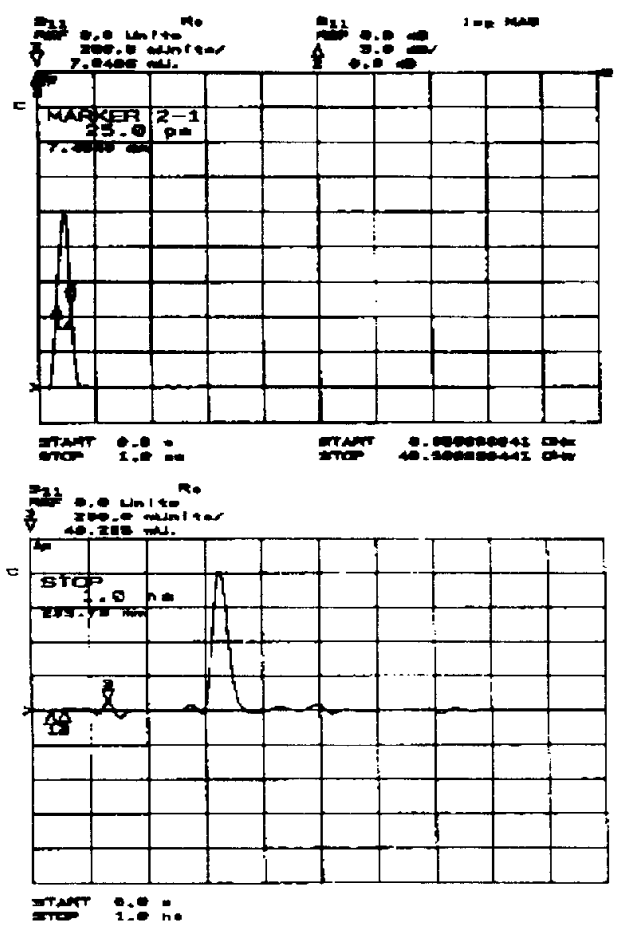

Fig.3. Examples of bench-test results. The top picture is the synthesized input signal, the bottom is the reflected signal from the pickup. (time-scale: $100 \mathrm{ps} / \mathrm{div}$ )

The wakefields, introduced into CTF acceleration sections by the very short bunches, escape and run through the smooth beam pipe of the pickup, forming a noisy background. Due to the cutoff frequency of the accelerator exit window, the escaping wakefields are at high frequency, and from the measurements they are above $6 \mathrm{GHz}$. There are two ways to process the signals. One way is to use analogue Gaussian filters to extract the low-frequency beam signal followed by further electronics to select the signals of each bunch. Another way is to use the wavelet transform to extract beam signals digitally out of the wakefield signal, and calculate the beam position using a computer.

\section{GAUSSIAN FILTER}

A bunch train signal can be considered as a series of single pulses. We are interested in recovering the signals of each bunch from a messy wakefield in order to measure each bunch's intensity and position. For this application, the behavioar of filters in the time domain and their transient response is more important and convenient for us. We do not need a very selective filter in the frequency domain, because we are not processing a single frequency as an ordinary BPM system does. An ideal Gaussian filter has the following response $^{[2 \mid}$ to an impulse at $t=0$ :

$$
f_{i}(t)=\frac{\Omega_{c}}{2 \sqrt{\pi \ln 2}} \exp -\left[\frac{\left(\Omega_{c} t-n \pi / 2\right)^{2}}{4 \ln 2}\right]
$$

Here $\Omega_{\mathrm{c}}$ is the frequency whose amplitude corresponds to the FWHM of the Gaussian filter in the frequency domain, $n$ is the number of cascaded stages, $t$ is time. This impulse response has two remarkable features:

1. The impulse response is identical in shape to the absolute magnitude of the frequency response.

2. No overshoot or ringing is exhibited, giving the Gaussian filter a localization feature in the time domain.

Because two adjacent bunches in the CTF are $330 \mathrm{ps}$ apart, a quasi-Gaussian filter (made by Picosecond Pulse Lab) with a rise time of 100 ps was selected, corresponding to a $3 \mathrm{~dB}$ bandwidth of $3.5 \mathrm{GHz}$. The filter is not ideal: although attenuation at $9 \mathrm{GHz}$ is $35 \mathrm{~dB}$, the attenuation at $16 \mathrm{GHz}$ is only $20 \mathrm{~dB}$. The second windows of Figs. 4 and 6 show the original signals picked up by the cone button monitor. Figs. 5 and 7 show the signals at the output of a Gaussian filter. Although there is some residual high frequency, the tests show that the Gaussian filter has effectively filtered out most of the wakefields.

One does not care about high-frequency resonances such as at $40 \mathrm{GHz}$ if the monitor is only working as a BPM with low frequency. However, when the monitor is used as a longitudinal pickup, it is useful to preserve the high-frequency components. Therefore it should have no ringing. Also, when a digital filter such as a wavelet transform is used, one would like to have a beam signal with all the bandwidth present.

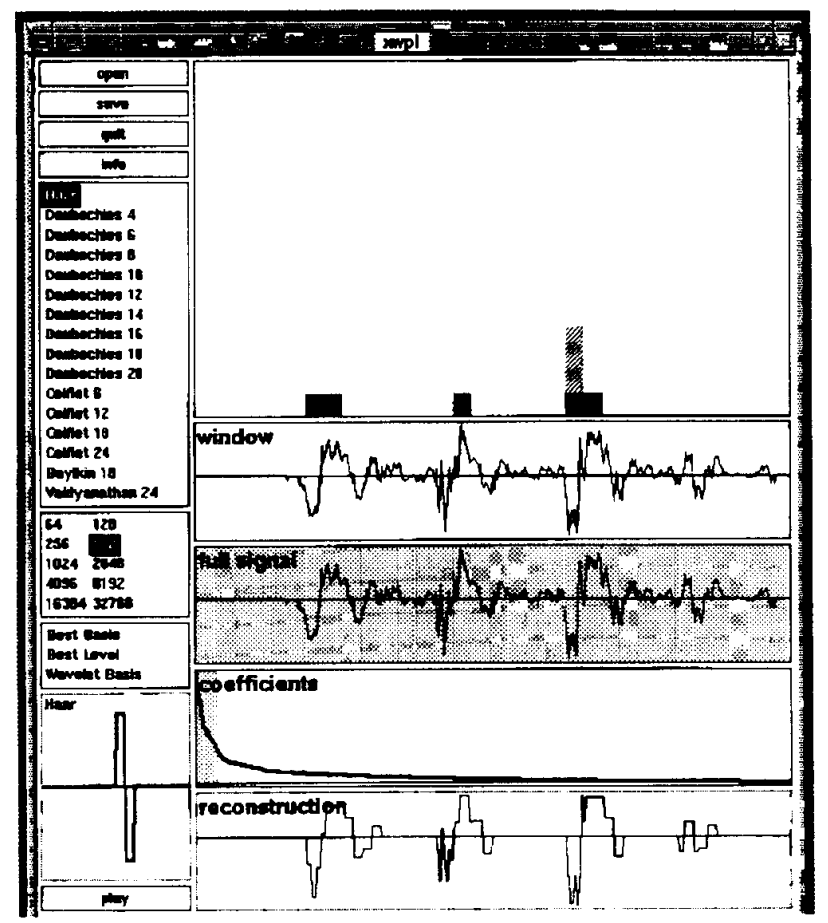

Fig.4 The original 3-bunch beam train signal from the pickup and the 3-bunch signal after the wavelet transform. (1.5 ns full width) 


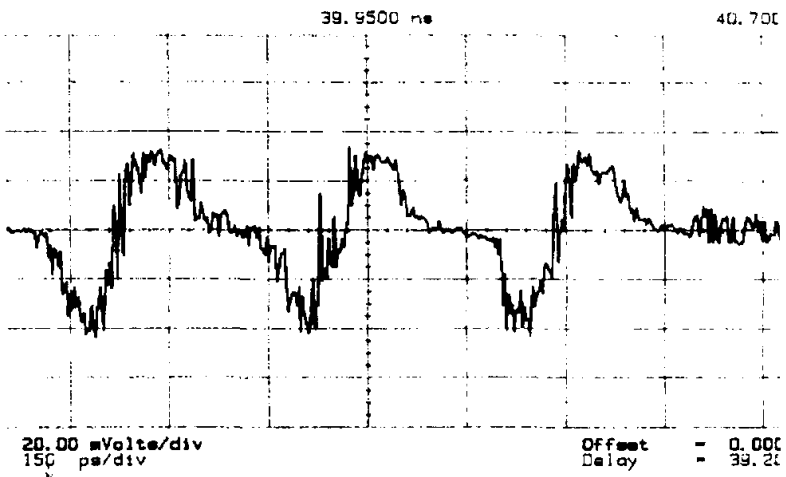

Fig.5 The 3-bunch beam train signal following the Gaussian filter.

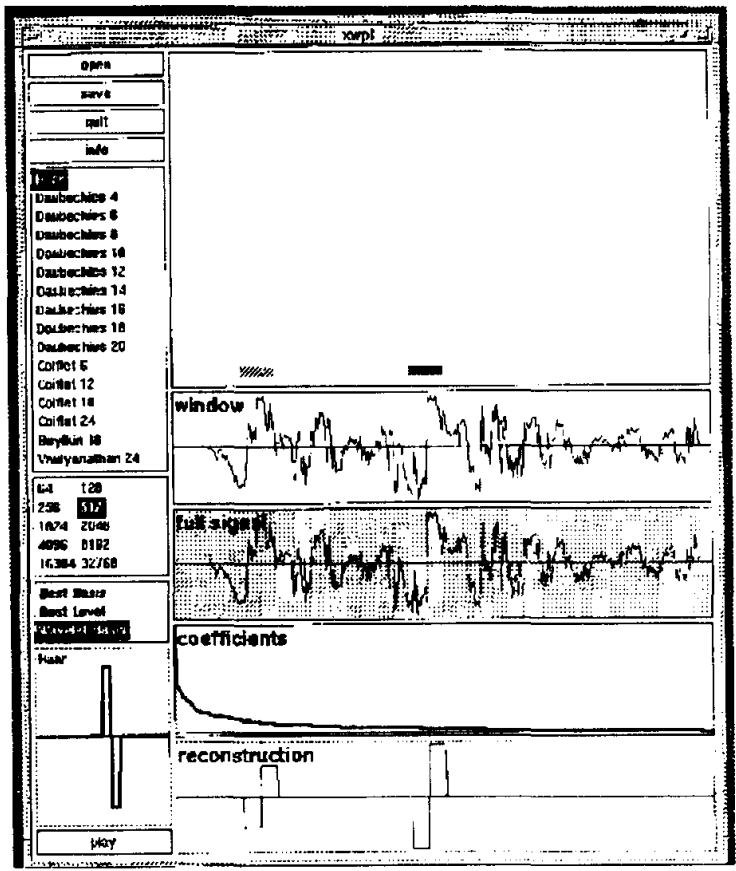

Fig. 6 The two-bunch beam train signals picked up by the cone button monitor and the signal extracted with the wavelet transform..(1 ns full width)

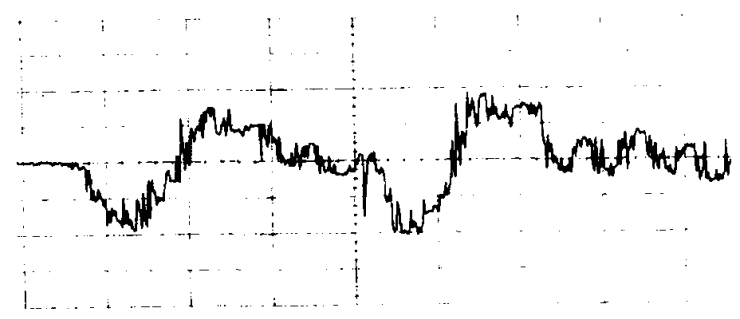

Fig.7 The two-bunch beam train signals at the output of a Gaussian filter. (100 ps/div)

\section{WAVELET TRANSFORM}

In principle, the wavelet transform is similar to the Fourier transform but with different base functions. The Fourier transform uses $\sin$ and $\cos$ as a set of base functions, but the wavelet tranform uses many other base functions. Because of its localization feature and the large selection of base functions, the wavelet transform can be used as a very good fast digital filter. Wavelet calculations have been done with the computer code XWPL ${ }^{[3]}$. The cone-shaped button pickup gives a differentiated beam signal. Among the wavelet base functions provided by XWPL, the Haar function has the greatest similarity to the signals, and has therefore been selected for our calculations.

The wavelet calculation picks the beam signals out of the wakefields and reveals the timing of the bunches as in Fig.4 and Fig.6, where the vertical axis is amplitude, and the horizontal axis is time. Although the beam shape is rough, its amplitude can still be used for position calculation due to the linearity of the transformation.

The fidelity of the wavelet-transformed signal depends on the number of coefficients used. In Fig.4, more coefficients are used than in Fig.6, so the transformed signals more closely approximate to the real beam signals. In Fig.6, only a very few coefficients are used in order to filter out the wakefields and the transformed beam signals become almost like the Haar function itself.

\section{CONCLUSION}

Both a Gaussian filter and wavelet transformation can recover certain parts of beam signals from a strong wakefield background. How close the recovered signals are to the real beam signal is still a subject for further study, but for beam position and intensity measurement they are adequate. The Gaussian filter works in a low-frequency range in order to filter out the high-frequency wakefields, but is a real-time analogue device, which is advantageous. On te other hand the wavelet transformation can extract beam signals over the whole measuring frequency range, but with the disadvantage that it is implemented as an off-line digital filter .

\section{ACKNOWLEDGMENT}

The authors thank Richard Lee (TRIUMF) for help with wavelet calculations, S.Koscielniak and B.Rawnsley for good discussions, and F.Caspers, Jean-Piere Delahaye (CERN) and many people at CLIC for their contributions and support with this project.

\section{REFERENCE}

[1].Yan Yin, Fritz Caspers, Elmar Schulte (CERN), Tord Ekelöf, Dag Reistad (TSL, Sweden), Design and Test Results of a Beam Monitor for the CERN Linear Collider Test Facility, BIW94, Oct. 1994, Vancouver, Canada.

[2]. Anatol I Zverev, Handbook of Filter Synthesis, John Wiley and Sons, Inc. New York, 1967.

[3]. Fazal Majid, et al. The XWPL system, (version 1.3, available by anonymous ftp from pascal.math.yale.edu (128.36.23.1) in the directory /pub/software/xwpl). 
